\title{
Efetividade da Alimentação na Prevenção do Câncer de Tireoide: Revisão Sistemática
}

doi: https://doi.org/10.32635/2176-9745.RBC.2020v66n4.1072

\author{
Effectiveness of Food in Preventing Thyroid Cancer: Systematic Review \\ Eficacia de los Alimentos en la Prevención del Cáncer de Tiroides: Revisión Sistemática
}

\section{Laura Faustino Gonçalves'; Cláudia Tiemi Mituuti²; Patrícia Haas ${ }^{3}$}

\section{RESUMO}

Introduçáo: A glândula tireoide sofre alteraçôes metabólicas que podem ocasionar doenças benignas ou malignas, sendo uma delas o câncer de tireoide, o qual está crescendo cada vez mais ao redor do mundo, sendo as mulheres mais propensas a desenvolver essa doença. Objetivo: Apresentar evidências científicas com base em revisão sistemática da literatura (PRISMA), verificando a efetividade da alimentação na prevenção do câncer de tireoide. Método: Para a seleção dos estudos, utilizou-se a combinação baseada no Medical Subject Heading Terms (MeSH). Foram utilizadas as bases de dados MEDLINE (PubMed), LILACS, SciELO, BIREME e Scopus. O período de busca dos artigos compreendeu entre janeiro de 2010 até março de 2020, sem restrição de idioma e localização. Resultados: Foram recuperados 32 artigos com potencial de inclusão, sendo que três responderam à pergunta norteadora que consistiu em analisar qual a efetividade da alimentação na prevençáo do câncer de tireoide. Conclusáo: Os estudos relataram uma possível associação entre a ingestão de alimentos e a prevençâo do câncer de tireoide. Sugere-se que os alimentos ricos em cálcio possam fornecer um papel protetor contra esse tipo de câncer, porém níveis excessivos de iodo na dieta também podem, de forma negativa, afetar a sua função da tireoide em razão das alteraçôes nos seus níveis de hormônio. As pesquisas demonstram também que as mulheres apresentam significativamente maior incidência de câncer de tireoide em relação aos homens.

Palavras-chave: Neoplasias da Glândula Tireoide/prevenção \& controle; Alimentos/efeitos adversos; Dieta Saudável; Revisão Sistemática.

\section{ABSTRACT}

Introduction: The thyroid gland undergoes metabolic changes that can cause benign or malignant diseases, one of which is thyroid cancer, which is increasing worldwide, and women are most prone to develop this disease. Objective: To present scientific evidences based on a systematic review of the literature (PRISMA) verifying the effectiveness of food in preventing thyroid cancer. Method: For the selection of studies, the combination based in the Medical Subject Heading Terms (MeSH) was used. The MEDLINE (PubMed), LILACS, SciELO, BIREME and Scopus databases were utilized. The search period for the articles ranged from January 2010 to March 2020, with no language or localization restrictions. Results: 32 articles with potential for inclusion were retrieved, and three articles responded to the guiding question that consisted in analyzing the effectiveness of food in preventing thyroid cancer. Conclusion: Studies have reported a possible association between food intake and the prevention of thyroid cancer. It is suggested that food rich in calcium may play a protective role against thyroid cancer, but excessive levels of iodine in the diet may also affect thyroid function negatively due to changes in hormone levels. The researches also demonstrate that women have significantly higher incidence of thyroid cancer than men.

Key words: Thyroid Neoplasms/prevention \& control; Food/adverse effects; Diet, Healthy; Systematic Review.

\section{RESUMEN}

Introducción: La glándula tiroides sufre cambios metabólicos que pueden causar enfermedades benignas o malignas, una de las cuales es el cáncer de tiroides. Lo cáncer de tiroides está creciendo cada vez más en todo el mundo, siendo las mujeres las más propensas a desarrollar esta enfermedad. Objetivo: Presentar evidencia científica basada en una revisión sistemática de la literatura (PRISMA) que verifique la efectividad de los alimentos en la prevención del cáncer de tiroides. Método: Para la selección de los estudios, se utilizó la combinación basada en los términos de encabezado de temas médicos (MeSH). Se utilizaron las bases de datos MEDLINE (PubMed), LILACS, SciELO, BIREME y Scopus. El período de búsqueda de los artículos varió desde enero de 2010 hasta marzo de 2020, sin restricciones de idioma y localización. Resultados: Se recuperaron 32 artículos con potencial de inclusión, y tres artículos respondieron a la pregunta guía que consistió en analizar la efectividad de los alimentos para prevenir lo cáncer de tiroides. Conclusión: Los estudios han reportado una posible asociación entre la ingesta de alimentos y la prevención de cáncer de tiroides. Se sugiere que los alimentos ricos en calcio pueden proporcionar un papel protector contra lo cáncer de tiroides, pero los niveles excesivos de yodo en la dieta también pueden afectar negativamente la función tiroidea debido a los cambios en los niveles hormonales. La investigación también muestra que las mujeres tienen una incidencia significativamente mayor de cáncer de tiroides en comparación con los hombres.

Palabras clave: Neoplasias de la Tiroides/prevención \& control; Alimentos/ efectos adversos; Dieta Saludable; Revisión Sistemática. 


\section{INTRODUÇÃO}

A tireoide é uma glândula endócrina localizada na base do pescoço, a qual possui a função de produção dos hormônios tireoidianos. Essa glândula sofre alteraçóes metabólicas que podem ocasionar doenças benignas ou malignas, sendo uma delas o câncer ${ }^{1}$.

Em 2018, houve 567 mil casos novos de câncer de tireoide no mundo, equivalente a $3 \%$ de todos os cânceres, ocupando a nona posição mundial. No mundo, cerca de 141 mil casos novos de câncer de tireoide e 35.575 óbitos foram registrados. No ano de 2018, no Brasil, ocorreram 748 óbitos por câncer de tireoide, sendo 239 em homens e $509 \mathrm{em}$ mulheres. As mulheres são mais propensas a desenvolver alterações, como nódulos, em virtude do estrogênio, um hormônio feminino que estimula a proliferação de células da tireoide. O câncer de tireoide corresponde a cerca de $1 \%$ de todas as neoplasias malignas, contudo, é o mais frequente dos tumores endócrinos malignos ${ }^{2}$.

O diagnóstico de câncer de tireoide pode ser realizado com o exame clínico na regiáo do pescoço, associado ao exame de ultrassonografia. Posteriormente, é realizada uma biópsia de uma amostra do tecido, o qual é examinado histologicamente, classificando o tumor como maligno ou benigno. Embora o índice de câncer de tireoide tenha aumentado relativamente, pouco se sabe efetivamente sobre a etiologia dessa doença. No entanto, diferenças nos índices de incidência internacionais de câncer de tireoide sugerem que fatores de estilo de vida ou ambientais, incluindo dieta, podem desempenhar um papel significativo no aparecimento do câncer de tireoide ${ }^{3}$.

Cordeiro e Martini ${ }^{4}$ relatam que fatores dietéticos estão relacionados significativamente com o aparecimento do câncer de tireoide. Entre os fatores alimentares, vegetais como couve, brócolis, couve-flor e mandioca, parecem estar relacionados à prevençáo do desenvolvimento de câncer de tireoide. Estudos epidemiológicos indicam que os hábitos alimentares podem desempenhar um papel relevante na proteção desse tipo de câncer. O iodo é o fator mais comumente investigado, por causa do seu papel na produção de hormônios tireoidianos na glândula tireoide e em seus distúrbios. Estudos epidemiológicos demonstram estreita relação entre câncer de tireoide, obesidade, estilo de vida e nutrição. Como medida de prevenção para esse tipo de doença, evidencia-se a importância da introdução de alimentos na dieta, que contenham propriedades antioxidantes, sendo preconizado o alto consumo de frutas e hortaliças ${ }^{5-8}$.

A presente pesquisa apresenta como objetivo principal e norteador verificar as evidências científicas sobre a efetividade da alimentaçấo na prevenção do câncer de tireoide, visando a responder à seguinte pergunta de pesquisa: Existe efetividade da alimentação na prevenção do câncer de tireoide?

\section{MÉTODO}

A revisão sistemática foi conduzida conforme as recomendaçôes do Preferred Reporting Items for Systematic Reviews and Meta-Analyses (PRISMA) ${ }^{9}$. A presente revisão sistemática foi registrada pelo número 190685 - PROSPERO (Centre for Reviews and Dissemination). As buscas por artigos científicos foram conduzidas por dois pesquisadores independentes nas bases de dados eletrônicas MEDLINE (PubMed) (https://www.ncbi. nlm.nih.gov/pubmed/), LILACS (http://lilacs.bvsalud. org/), SciELO (http://www. scielo.br/), BIREME (https://bvsalud.org/) e Scopus (https://www.scopus. com) sem restrição de idioma, período e localização. A pesquisa foi estruturada e organizada na forma PICOS, que representa um acrônimo para População-alvo, a Intervenção, Controle, "Outcomes", "Study" (Quadro 1). Considerando o objetivo desta pesquisa, o acrômio Controle não foi utilizado, por não ser aplicável.

Quadro 1. Descrição dos componentes do acrônimo PICOS

\begin{tabular}{|l|l|}
\hline \multicolumn{1}{|c|}{ Acrônimo } & \multicolumn{1}{c|}{ Definição } \\
\hline P & Pacientes \\
\hline $\mathbf{I}$ & Alimentação \\
\hline $\mathbf{C}$ & Não aplicável \\
\hline $\mathbf{O}$ & Câncer de tireoide \\
\hline $\mathbf{S}$ & $\begin{array}{l}\text { Estudo descritivo } \\
\text { Estudo transversal } \\
\text { Estudo observacional }\end{array}$ \\
\hline
\end{tabular}

Os descritores foram selecionados a partir do dicionário Descritores em Ciências da Saúde (DeCS) e Medical Subject Heading Terms (MeSH). Foram propostos para as buscas os seguintes descritores e operadores boleanos: (thyroid cancer) and (food) and (protective effect) AND (randomized controlled trial[pt] OR controlled clinical trial[pt] OR randomized controlled trials[mh] OR random allocation [mh] OR double-blind method[mh] OR singleblind method[mh] OR clinical trial[pt] OR clinical trials[mh] OR (“clinical trial” $[\mathrm{tw}])$ OR ((singl*[tw] OR doubl*[tw] OR trebl*[tw] OR tripl*[tw]) AND (mask* $\mathrm{tw}$ OR blind*[tw])) OR ("latin square" [tw]) OR placebos[mh] OR placebo*[tw] OR random*[tw] OR research design[mh:noexp] OR follow-up studies[mh] OR prospective studies[mh] OR cross-over studies[mh] 
OR control*[tw] OR prospectiv* ${ }^{*}[\mathrm{tw}]$ OR volunteer*[tw]) NOT (animal[mh] NOT human[mh]).

Os desenhos dos estudos selecionados consistiram em estudo descritivo, estudo transversal, estudo de coorte e estudo de caso, e somente com humanos. Foram incluídos estudos sem restrição de idioma e localização, no período de janeiro de 2010 até março de 2020, para busca dos artigos. O Quadro 2 representa os critérios de inclusão e exclusão desenvolvidos nesta pesquisa. Todos os artigos analisados foram nos idiomas de português, inglês, francês e espanhol; dessa forma, os autores não apresentaram dificuldades nas suas traduçóes.

Quadro 2. Síntese dos critérios de inclusão/exclusão

\begin{tabular}{|c|c|}
\hline \multicolumn{2}{|c|}{ Critérios de inclusão } \\
\hline Delineamento & $\begin{array}{l}\text { Relatos de casos } \\
\text { Estudos de casos e controle } \\
\text { Ensaios clínicos controlados } \\
\text { Estudos de coorte } \\
\text { Estudos em triagem } \\
\text { Estudos observacionais }\end{array}$ \\
\hline Localização & Sem restrição \\
\hline Idioma & Sem restrição \\
\hline \multicolumn{2}{|c|}{ Critérios de exclusão } \\
\hline Delineamento & $\begin{array}{l}\text { Cartas ao editor } \\
\text { Diretrizes } \\
\text { Revisões de literatura } \\
\text { Revisões sistemáticas } \\
\text { Meta-análises }\end{array}$ \\
\hline Estudos & $\begin{array}{l}\text { Estudos pouco claros, } \\
\text { mal descritos ou } \\
\text { inadequados }\end{array}$ \\
\hline $\begin{array}{l}\text { Forma de } \\
\text { publicação }\end{array}$ & Apenas resumo \\
\hline
\end{tabular}

Foram excluídos estudos publicados no formato de cartas ao editor, diretrizes, revisôes de literatura, revisôes sistemáticas, meta-análises e resumos. Estudos pouco claros, com informações insuficientes ou indisponíveis.

A extraçáo dos dados para o processo de elegibilidade dos estudos foi realizada utilizando-se uma ficha própria para revisão sistemática elaborada pelos pesquisadores em Programa Excel, no qual os dados extraídos foram adicionados inicialmente por um dos pesquisadores e, então, conferidos pelo outro pesquisador. Para os dados obtidos dos estudos elegíveis, estes também foram transportados para uma planilha em mesmo programa, a fim de organizar os resultados conforme descrito no Quadro 3.

\section{RESULTADOS}

Foram identificados 32 artigos inicialmente; dos quais, oito foram para a avaliação de resumos. Destes, dois foram excluídos por não responderem à pergunta norteadora. Procedeu-se à leitura na íntegra dos cinco artigos e, após a aplicação dos critérios de elegibilidade, três estudos ${ }^{1,10,11}$ sobre alimentação e prevenção do câncer de tireoide foram objeto da presente análise (Figura 1). Os estudos analisados foram do tipo estudo caso-controle e transversal. $\mathrm{Na}$ análise, foram categorizados, segundo o tema investigado, perfil alimentar de sujeitos diagnosticados com câncer de tireoide, alimentos de risco para o desenvolvimento de câncer de tireoide e alimentos que diminuem o risco de desenvolver o câncer de tireoide.

No estudo realizado por Cléro et al. ${ }^{1}$, cerca de $90 \%$ dos casos de câncer de tireoide eram em mulheres, $80 \%$ dos participantes com câncer de tireoide e $60 \%$ dos casos-controle estavam com sobrepeso ou obesidade (índice de massa corporal - IMC $\geq 25 \mathrm{~kg} / \mathrm{m}^{2}$ ), respectivamente. O estudo objetivou comparar a dieta ocidental com a dieta da Polinésia Francesa e não encontraram associação entre um padráo alimentar ocidental e o risco de câncer de tireoide, porém um padrão alimentar polinésio tradicional indicou um considerável risco de desenvolver esse câncer.

$\mathrm{Na}$ pesquisa de Cho et al. ${ }^{10}$, os participantes foram questionados sobre sua frequência de ingestão e o tamanho da porção de alimentos específicos consumidos durante o ano anterior, usando o questionário de frequência alimentar validado (QFA), que abrange 106 itens alimentares. Categorias de frequência (ou seja, nunca ou raramente, uma vez por mês, duas ou três vezes por mês) e três tamanhos de porção (pequenos, médios e grandes) são avaliados no QFA. A ingestão de nutrientes (por exemplo, macronutrientes, vitaminas e minerais) foi calculada multiplicando a frequência de consumo de cada unidade de alimento pelo conteúdo de nutrientes das porçôes especificadas. A ingestáo de proteína, gordura, cálcio e ferro na dieta foi calculada separadamente por duas fontes (animal ou vegetal). Obtiveram como resultado um alto consumo de cálcio associado a um risco reduzido de câncer de tireoide, porém não observaram associaçôes significativas entre o risco de câncer de tireoide e os outros nutrientes.

Destaca-se que Haslam et al. ${ }^{11}$, por meio de um questionário de base, analisaram dados demográficos, estado de saúde e consumo de peixe e obtiveram que os casos-controle (108 participantes) relataram um consumo médio mensal mais alto de peixes; além disso, os casos-controle foram mais propensos a ter uma alta ingestão a longo prazo de ômega-3 em comparação com os casos de pacientes com câncer de tireoide. A ingestão de ômega-3 foi associada a uma menor chance de desenvolvimento 
Quadro 3. Síntese dos artigos incluídos

\begin{tabular}{|c|c|c|c|c|c|c|c|}
\hline $\begin{array}{l}\text { Autor/ } \\
\text { Ano/ } \\
\text { Local }\end{array}$ & $\begin{array}{l}\text { Tipo de } \\
\text { estudo }\end{array}$ & $\begin{array}{c}\text { Sujeitos } \\
\text { participantes }\end{array}$ & $\begin{array}{l}\text { Faixa } \\
\text { etária }\end{array}$ & Objetivo & Exame & Resultados & Conclusão \\
\hline $\begin{array}{l}\text { Cléro; } \\
\text { Doyon; } \\
\text { Chungue et } \\
\text { al., } 2012 \\
\text { Polinésia } \\
\text { Francesa }\end{array}$ & Caso-controle & $\begin{array}{l}229 \text { sujeitos com } \\
\text { câncer de tireoide } \\
\text { diagnosticados } \\
\text { entre } 1979 \text { e } \\
2004 \text { (caso); } 371 \\
\text { participantes } \\
\text { selecionados } \\
\text { aleatoriamente } \\
\text { (controle) }\end{array}$ & $\begin{array}{l}56 \text { e } 62 \\
\text { anos }\end{array}$ & $\begin{array}{l}\text { Identificar padrões } \\
\text { alimentares } \\
\text { e investigar } \\
\text { a associação } \\
\text { entre padrões } \\
\text { alimentares ou } \\
\text { itens alimentares } \\
\text { e risco de câncer } \\
\text { de tireoide entre } \\
\text { a alimentação } \\
\text { tradicional da } \\
\text { Polinésia e a } \\
\text { alimentação } \\
\text { ocidental }\end{array}$ & $\begin{array}{l}\text { Questionário } \\
\text { adaptado } \\
\text { do European } \\
\text { Prospective } \\
\text { Investigation } \\
\text { in Cancer and } \\
\text { Nutrition (EPIC) }\end{array}$ & $\begin{array}{l}90 \% \text { dos casos de } \\
\text { câncer de tireoide eram } \\
\text { mulheres } \\
80 \% \text { dos participantes } \\
\text { e } 60 \% \text { dos controles } \\
\text { estavam com sobrepeso } \\
\text { ou obesidade } \\
\text { (IMC } \geq 25 \mathrm{~kg} / \mathrm{m}^{2} \text { ) } \\
0 \text { padrão ocidental não } \\
\text { foi associado ao risco de } \\
\text { câncer de tireoide } \\
0 \text { consumo de mandioca } \\
\text { foi significativamente } \\
\text { associado a uma } \\
\text { diminuiç̧áo do risco de } \\
\text { câncer de tireoide }\end{array}$ & $\begin{array}{l}\text { Um padrão alimentar } \\
\text { polinésio tradicional } \\
\text { levou a um } \\
\text { significativo risco de } \\
\text { desenvolvimento de } \\
\text { câncer de tireoide }\end{array}$ \\
\hline $\begin{array}{l}\text { Haslam; } \\
\text { Robb; } \\
\text { Bonner et } \\
\text { al., } 2016 \\
\text { EUA }\end{array}$ & $\begin{array}{l}\text { Coorte } \\
\text { longitudinal }\end{array}$ & $\begin{array}{l}27 \text { sujeitos com } \\
\text { câncer de tireoide } \\
\text { e } 108 \text { participantes } \\
\text { selecionados } \\
\text { aleatoriamente }\end{array}$ & $\begin{array}{l}\text { Média de } \\
32 \text { anos }\end{array}$ & $\begin{array}{l}\text { Examinar os } \\
\text { efeitos do consumo } \\
\text { de peixes no } \\
\text { Lago Ontário e o } \\
\text { consumo estimado } \\
\text { de PCB e ômega-3 } \\
\text { com o risco de } \\
\text { desenvolvimento } \\
\text { do câncer de } \\
\text { tireoide em } \\
\text { um grupo de } \\
\text { pescadores } \\
\text { diagnosticados com } \\
\text { câncer de tireoide } \\
\text { entre } 1^{\circ} \text { de junho } \\
\text { de } 1991 \text { e } 31 \text { de } \\
\text { dezembro de } 2008\end{array}$ & $\begin{array}{l}\text { Questionário de } \\
\text { acompanhamento }\end{array}$ & $\begin{array}{l}\text { Os resultados não indicam } \\
\text { associações significativas } \\
\text { entre o consumo de peixes } \\
\text { e ômega-3, estimados a } \\
\text { curto prazo ou o consumo } \\
\text { estimado de PCB dos } \\
\text { peixes dos Grandes Lagos } \\
\text { e o desenvolvimento } \\
\text { de câncer de tireoide, } \\
\text { mas foi sugerido que o } \\
\text { ômega-3 a longo prazo } \\
\text { dos peixes dos Grandes } \\
\text { Lagos pode proteger } \\
\text { o desenvolvimento do } \\
\text { câncer de tireoide }\end{array}$ & $\begin{array}{l}0 \text { consumo de peixe, } \\
\text { com os possíveis PCB } \\
\text { concomitantes, dos } \\
\text { Grandes Lagos, não } \\
\text { parece aumentar } 0 \\
\text { risco de câncer de } \\
\text { tireoide }\end{array}$ \\
\hline $\begin{array}{l}\text { Cho; Lee; } \\
\text { Kim, } 2016 \\
\text { Coreia }\end{array}$ & Caso-controle & $\begin{array}{l}113 \text { mulheres com } \\
\text { câncer de tireoide } \\
\text { e } 226 \text { mulheres } \\
\text { participantes } \\
\text { selecionadas }\end{array}$ & $\begin{array}{l}30 \text { anos } \\
\text { ou mais }\end{array}$ & $\begin{array}{l}\text { Investigar o papel } \\
\text { dos nutrientes no } \\
\text { risco de câncer } \\
\text { de tireoide em } \\
\text { mulheres coreanas } \\
\text { entre outubro de } \\
2007 \text { e julho de } \\
2014\end{array}$ & $\begin{array}{l}\text { Questionário } \\
\text { autoaplicável e } \\
\text { questionário de } \\
\text { QFA }\end{array}$ & $\begin{array}{l}\text { Descobriu-se que a } \\
\text { alta ingestão de cálcio } \\
\text { estava associada a um } \\
\text { risco reduzido de câncer } \\
\text { de tireoide. Associações } \\
\text { significativas foram } \\
\text { observadas entre } \\
\text { indivíduos com mais de } \\
50 \text { anos, baixo IMC e } \\
\text { baixa ingestão calórica. } \\
\text { No entanto, outros } \\
\text { nutrientes incluídos neste } \\
\text { estudo não mostraram } \\
\text { associações significativas } \\
\text { com o risco de câncer de } \\
\text { tireoide }\end{array}$ & $\begin{array}{l}\text { Possível efeito } \\
\text { protetor do } \\
\text { cálcio no risco de } \\
\text { desenvolvimento do } \\
\text { câncer de tireoide }\end{array}$ \\
\hline
\end{tabular}




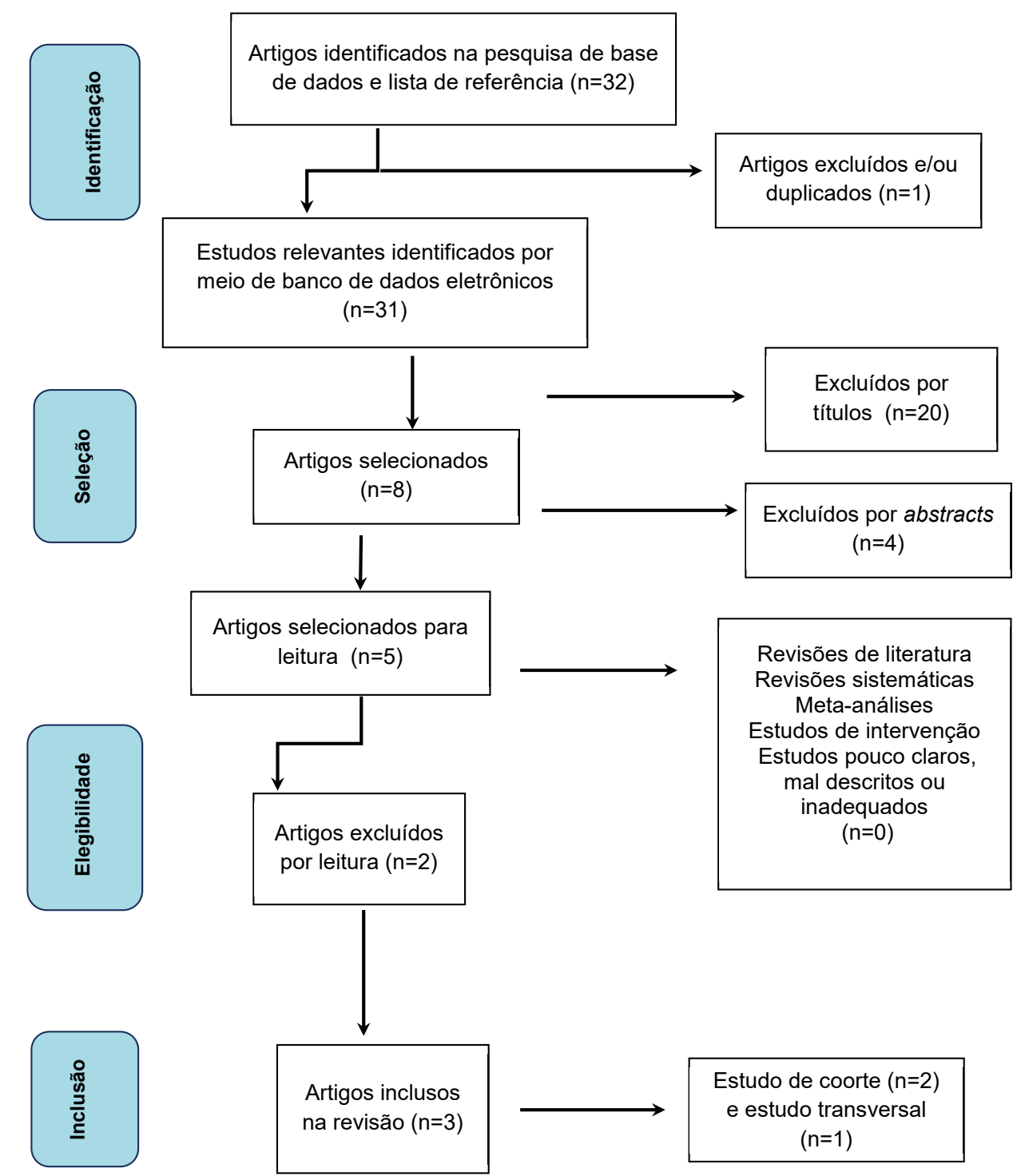

Figura 1. Fluxograma de busca e análise dos artigos

de câncer de tireoide. Além disso, a ingestáo de bifenilos policlorados (PCB); ou seja, compostos orgânicos sintéticos, não foi significativamente associada ao desenvolvimento de câncer de tireoide.

\section{DISCUSSÃO}

Pesquisas determinaram alguns fatores de risco relevantes para o câncer de tireoide, com exposição em diferentes padróes alimentares, estilos de vida, nutrição ou outros fatores de risco ambiental em populaçóes distintas ${ }^{12,13}$. Alguns estudos relatam que os fatores alimentares como produtos processados e industrializados de uma maneira mais abrangente podem desempenhar um papel significativo na causa do câncer de tireoide, possivelmente influenciando os hormônios da tireoide e afetando a sua função ${ }^{14,15}$.
O estudo de Cléro et al. ${ }^{1}$ incluiu na pesquisa 24 alimentos ou grupos de alimentos na análise como tubérculos, cereais, alimentos ricos em amido, repolho, legumes, frutas cítricas, banana, manga, mamão, goiaba, maracujá, melancia, maçá, pera, pêssego, damasco, água de coco, nozes, peixe do oceano, peixe da lagoa, marisco, carne, carne de porco cozida, produtos lácteos, ovos, comida chinesa, bolos, chá, café, bebidas doces e alcoólicas. Os autores usaram tabelas de composição de alimentos para converter alimentos em nutrientes. Para cada indivíduo, calcularam também a ingestâo média diária de energia na dieta. Os autores estabeleceram em sua pesquisa dois padrốes de dieta, o primeiro foi denominado padrão ocidental, no qual os participantes apresentavam em sua dieta carne vermelha, carne de porco cozida, ovos, comida chinesa, cereais (macarrão, arroz e pão), alimentos ricos em amido como batatas e leguminosas, 
repolho e outros vegetais, bolos, bebidas doces, bebidas alcoólicas e café.

O segundo padrão apresentado por Cléro et al. ${ }^{1}$ foi rotulado de polinésio tradicional, o qual foi caracterizado por um alto consumo de peixe (lagos e oceano), mariscos, banana e frutas cítricas, manga, papaia, goiaba, água de coco, fruta-pão, tubérculos (mandioca, batata-doce) e laticínios. Como resultado, obteve que a dieta ocidental não se associou significativamente ao risco de câncer de tireoide e ao padrão alimentar polinésio tradicional, porém uma reduçáo significativa do risco de câncer de tireoide foi observada com o consumo de mandioca, mas não com o consumo de repolho, por exemplo.

Segundo a literatura, as principais formas de evitar o câncer é ingerir uma alimentação saudável, praticar atividade física e manter o peso corporal adequado. Ingestão de alimentos ricos em origem vegetal como frutas, legumes, verduras, cereais integrais, feijóes e outras leguminosas e uma alimentação pobre em alimentos ultraprocessados e bebidas açucaradas podem prevenir novos casos de câncer ${ }^{16}$. A pesquisa de Baenal ${ }^{17}$ cita que dietas à base de vegetais, como frutas, verduras, leguminosas e grãos integrais, os quais contêm grande quantidade de fitoquímicos bioativos, tendem a reduzir o risco de doenças crônicas. Já o aumento do consumo de peixes está associado com menor mortalidade e morbidade cardiovascular, além de efeitos positivos no metabolismo das lipoproteínas, coagulação e função das plaquetas, função endotelial e rigidez arterial $^{18}$.

O estudo de caso-controle realizado por Przybylik-Mazurek et al. ${ }^{19}$ com aplicação de questionário incluía informaçôes sobre padróes alimentares. Nele, havia 284 pacientes com câncer de tireoide (grupo caso), sendo 30 homens com idade média de 58 anos de idade e 254 mulheres com idade média de 52 anos de idade; e 345 participantes sem câncer de tireoide como controle, os quais foram selecionados aleatoriamente (58 homens de idade média de 60 anos de idade e 287 mulheres com idade média de 53 anos de idade) e avaliados. Os participantes selecionados de forma aleatória foram pareados por idade e sexo com o grupo que apresentava câncer de tireoide. Foram analisados os principais grupos de produtos nutricionais; ou seja, alimentos ricos em amido, carne, laticínios, vegetais, frutas e bebidas. Como resultado, observaram que o consumo de vegetais, frutas, peixe de água salgada e queijo foi significativamente menor em pacientes com câncer de tireoide do que nos participantes sem diagnóstico de câncer de tireoide. Os autores concluíram que os padrôes alimentares parecem modificar o risco de desenvolvimento do câncer de tireoide. Uma dieta rica em vegetais e frutas, bem como peixe de água salgada e carne com baixo teor de gordura, pode ser relatada como um importante fator de proteção.
Schwingshackl e Hoffmann ${ }^{20}$ citam que a dieta mediterrânica se caracteriza por conter alimentos antioxidantes e anti-inflamatórios. Essa dieta está relacionada, segundo pesquisas, à prevenção do envelhecimento celular e de várias doenças crónicas. A dieta mediterrânica é caracterizada pelo consumo de frutas frescas, oleaginosas, leguminosas e cereais. A literatura refere que a incidência de câncer geral é menor nos países que fazem fronteira com o Mar Mediterrâneo e relatam que a adesão a um padrão alimentar mediterrânico pode ser correlacionada com um risco reduzido de vários tipos de câncer, além da redução de mortalidade por câncer ${ }^{21}$.

Cho et al. ${ }^{10}$ categorizaram os participantes em dois grupos de acordo com seus níveis de ingestáo de cálcio e examinaram se as características gerais dos participantes diferiam dos níveis de ingestão de cálcio. As características gerais dos participantes não foram significativamente diferentes nos grupos com alto e baixo consumo de cálcio. Para examinar os efeitos de outros fatores de risco na associação entre ingestão de cálcio e câncer de tireoide, os pesquisadores realizaram a análise de subgrupos por idade, IMC, tabagismo e nível de ingestão calórica total. Associaçóes significativas foram observadas em indivíduos com mais de 50 anos e que apresentaram baixa ingestão calórica. No entanto, essas associaçōes não foram observadas em uma análise de subgrupo que diferenciava a fonte de cálcio (planta ou animal).

Já Liang et al. ${ }^{12}$, no estudo de caso-controle, examinaram a associação entre padrão alimentar e risco de câncer de tireoide no período de 2010 e 2011 . O estudo incluiu 390 casos de câncer de tireoide e 436 indivíduos sem câncer de tireoide. Os participantes responderam a questionários de histórico alimentar de base (DHQ), nos quais três padróes alimentares foram presenciados de acordo com os principais alimentos consumidos pelos participantes: dieta com prevalência de alimentos com amido e produtores açucarados; dieta à base de frutas e legumes; e dieta com prevalência de proteína e gordura. Os achados mostram que a dieta com predomínio de frutas e vegetais foi significativamente associada a um risco reduzido de câncer de tireoide. Quando comparadas às mulheres mais jovens, foi observado um efeito protetor maior em mulheres com idade $\geq 50$ anos em relação ao risco de desenvolver o câncer de tireoide. Dieta rica em alimentos com amido mostrou-se efetiva na proteçáo ao câncer de tireoide, contudo, dietas nas quais prevalecem alimentos açucarados demonstraram não ser efetivas na proteção ao câncer de tireoide.

No entanto, Haslam et al. ${ }^{11}$ em seus resultados mostraram que não houve associaçóes significativas entre o consumo de peixes e o desenvolvimento de câncer de tireoide. $\mathrm{O}$ consumo de compostos químicos como $\mathrm{PCB}$, 
presente nos peixes, não foi significativamente associado ao desenvolvimento de câncer de tireoide. Relatam também a possibilidade de que as concentraçóes de $\mathrm{PCB}$ nos peixes não alterem as concentraçôes hormonais da tireoide e, portanto, não aumentariam a incidência de câncer. Os autores relatam não haver um risco de desenvolver câncer de tireoide entre os pescadores que têm uma maior exposição ao composto $\mathrm{PCB}$ dos peixes, em comparação com aqueles que têm uma menor exposição estimada ao PCB. No entanto, a ingestáo prolongada de ômega-3 parece ser protetora com relação ao desenvolvimento de câncer de tireoide.

Zamora-Ros et al..$^{15}$, em seu estudo de coorte com 748 pacientes de câncer de tireoide (666 mulheres e 601 do tipo papilar), com idade entre 35 e 70 anos, objetivaram relacionar o consumo de peixes e o desenvolvimento de câncer de tireoide. Os dados sobre a ingestão de peixe magro, peixe gordo, produtos de peixe e mariscos foram coletados usando-se questionários alimentares validados. $\mathrm{O}$ estudo mostrou que a ingestáo de peixes e moluscos não estava associada ao risco diferenciado de câncer de tireoide. Zamora-Ros et al. ${ }^{15}$ realizaram um estudo de coorte com o objetivo de examinar a associação entre o consumo de frutas, vegetais e sucos de frutas com o risco de desenvolver câncer de tireoide. Os participantes foram recrutados entre 1991 e 2000 e a ingestão de frutas e legumes e suco de frutas foi avaliada por meio de questionários validados. Os autores não encontraram associação significativa entre a ingestáo de frutas e verduras e o risco de desenvolver câncer de tireoide, no entanto, observou-se uma tendência positiva de proteção com a ingestão de suco de frutas.

Em contrapartida, Jung et al. ${ }^{13}$, usando um questionário quantitativo com 121 itens, objetivaram avaliar a associaçáo entre a ingestáo de frutas e vegetais crus e o câncer de tireoide em um estudo caso-controle, e observaram 111 casos de câncer de tireoide nos quais a ingestáo de alimentos e nutrientes foi estimada. Como resultado, os autores relataram que o alto consumo de vegetais crus, caquis e tangerinas pode diminuir o risco de câncer de tireoide e ajudar a prevenir esse câncer em estágio inicial.

Cho et al. ${ }^{10}$ pesquisaram a alta ingestão de cálcio, que parece estar associada a um risco reduzido de câncer de tireoide. Associaçóes significativas entre o desenvolvimento do câncer de tireoide foram observadas entre indivíduos com mais de 50 anos, baixo IMC e baixa ingestáo calórica. No entanto, outros nutrientes incluídos neste estudo não mostraram associaçôes significativas com o risco de câncer de tireoide. Portanto, os autores relatam um possível efeito protetor do cálcio no risco de desenvolvimento do câncer de tireoide.
A pesquisa de Bandurska-Stankiewicz et al. ${ }^{14}$ indica que realizaram um estudo com o objetivo de investigar os papéis dos hábitos e vícios nutricionais na incidência de câncer de tireoide com 297 pacientes com câncer de tireoide e 589 saudáveis, dos quais, $46 \%$ dos pacientes (totalidade) declararam consumo frequente de leite e derivados. Os pacientes com câncer de tireoide consumiram plantas crucíferas (ricas em antioxidantes, vitaminas, fibras e minerais), significativamente mais frequente do que indivíduos saudáveis, enquanto o consumo de ovos entre os dois grupos de pacientes foi semelhante. O baixo consumo de peixe e o consumo médio de sal de mesa foram pareados em ambos os grupos. A pesquisa mostrou que o risco de câncer de tireoide aumentou em pacientes que consumiram vegetais crucíferos na província de Olsztyn, Polônia. Sua ingestão regular foi relacionada a 1,5 vezes maior risco de incidência de câncer de tireoide.

\section{CONCLUSÃO}

Os dados encontrados a partir desta revisão sistemática evidenciaram que um alto consumo de cálcio parece estar associado a um risco reduzido de câncer de tireoide. Além disso, os autores corroboram que a ingestão prolongada de ômega-3 parece ser protetora para o desenvolvimento de câncer de tireoide. As análises demonstram também que mulheres apresentam significativamente maior probabilidade de desenvolver câncer de tireoide em relação aos homens. A produção científica sobre esse tema ainda é incipiente e o avanço nessa investigação é fundamental, principalmente considerando diferentes populaçóes, as quais possuem padróes alimentares e estilos de vida diversos, além de estarem expostas a fatores ambientais distintos.

\section{CONTRIBUIÇÕES}

Laura Faustino Gonçalves e Cláudia Tiemi Mituuti contribuíram na concepção e/ou no planejamento do estudo; e na obtenção, análise e interpretação dos dados. Patrícia Haas contribuiu na concepção e/ou no planejamento do estudo; na obtençáo, análise e interpretaçáo dos dados; assim como na redação e revisão crítica. Todas as autoras aprovaram a versão final a ser publicada.

\section{DECLARAÇÃO DE CONFLITO DE INTERESSES}

Nada a declarar.

\section{FONTES DE FINANCIAMENTO}

Não há. 


\section{REFERÊNCIAS}

1. Cléro É, Doyon F, Chungue V, et al. Dietary iodine and thyroid cancer risk in French Polynesia: a case - control study. Thyroid. 2012;22(4):422-9. doi: https://doi. org/10.1089/thy.2011.0173

2. Instituto Nacional de Câncer José Alencar Gomes da Silva [Internet]. Rio de Janeiro: INCA; [data desconhecida]. Câncer: tipos de câncer; 2019 [modificado 2019 set 12; acesso 2020 abr 5]. Disponível em: https://www.inca. gov.br/tipos-de-cancer

3. Pellegriti G, Frasca F, Regalbuto C, et al. Worldwide Increasing Incidence of Thyroid Cancer: update on epidemiology and risk factors. J Cancer Epidemiol. 2013;2013:965212. doi: https://doi. org/10.1155/2013/965212

4. Cordeiro EAK, Martini JG. Perfil dos pacientes com câncer de tireóide submetidos à radioiodoterapia. Texto Contexto Enferm. 2013;22(4):1007-14. doi: https:/doi. org/10.1590/S0104-07072013000400017

5. Carvalho GA, Perez CLS, Ward LS. Utilização dos testes de funçáo tireoidiana na prática clínica. Arq Bras Endocrinol Metab. 2013;57(3):193-204. doi: https://doi. org/10.1590/S0004-27302013000300005

6. Brenta G, Vaisman M, Sgarbi JA, et al. Clinical practice guidelines for the management of hypothyroidism. Arq Bras Endocrinol Metab. 2013;57(4):265-91. doi: https://doi.org/10.1590/ S0004-27302013000400003

7. Marini H, Polito F, Adamo EB, et al. Update on genistein and thyroid: an overall message of safety. Front Endocrinol (Lausanne). 2012;3:94. doi: https://doi. org/10.3389/fendo.2012.00094

8. Gar-Elnabi MEM, Taha RM, Omer MA, et al. Assessment of human thyroid function using radioimmunoassay and enzyme-linked-immuno-sorbent-assay. J Exp Clin Med. 2013;30(4): 317-21. doi: https://doi.org/10.5835/jecm. omu.30.04.007

9. Moher D, Shamseer L, Clarke M, et al. Preferred reporting items for systematic review and meta-analysis protocols (PRISMA-P) 2015 statement. Syst Rev. 2015;4(1):1. doi: https://doi.org/10.1186/2046-4053-4-1

10. Cho YA, Lee J, Kim J. Association between nutrient intake and thyroid cancer risk in Korean women. Nutr Res Pract. 2016 Jun;10(3):336-41. doi: https://doi. org/10.4162/nrp.2016.10.3.336

11. Haslam A, Robb SW, Bonner MR, et al. Polychlorinated biphenyls and omega-3 fatty acid exposure from fish consumption, and thyroid cancer among New York anglers. J Environ Sci (China). 2016;41:270-77. doi: https://doi.org/10.1016/j.jes.2015.05.004

12. Liang J, Zhao N, Zhu C, et al. Dietary patterns and thyroid cancer risk: a population-based case-control study. Am J Transl Res. 2020;12(1):180-90.
13. Jung SK, Kim K, Tae K, et al. The effect of raw vegetable and fruit intake on thyroid cancer risk among women: a case-control study in South Korea. Br J Nutr. 2013;109(1):118-28. doi: https://doi.org/10.1017/ S0007114512000591

14. Bandurska-Stankiewicz E, Aksamit-Białoszewska E, Rutkowska J, et al. The effect of nutritional habits and addictions on the incidence of thyroid carcinoma in the Olsztyn province of Poland. Endokrynol Pol. 2011;62(2):145-50.

15.Zamora-Ros R, Castañeda J, Rinaldi S, et al. Consumption of Fish Is Not Associated with Risk of Differentiated Thyroid Carcinoma in the European Prospective Investigation into Cancer and Nutrition (EPIC) Study. J Nutr. 2017;147(7):1366-73. doi: https:// doi.org/10.3945/jn.117.247874

16. Instituto Nacional de Câncer José Alencar Gomes da Silva[Internet]. Rio de Janeiro: INCA; [data desconhecida]. Causas e prevenção: alimentação; 2019. [modificado 2019 nov 21; acesso 2020 abr 25]. Disponível em: https:/www.inca.gov.br/alimentacao

17. Baenal RC. Muito além dos nutrientes: o papel dos fitoquímicos nos alimentos integrais. Diagn Tratamento. 2015; 20(1):17-21.

18. Scherr C, Gagliardi ACM, Miname MH, et al. Fatty acid and cholesterol concentrations in usually consumed fish in Brazil. Arq Bras Cardiol. 2014;104(2):152-8. doi: https://doi.org/10.5935/abc.20140176

19. Przybylik-Mazurek E, Hubalewska-Dydejczyk A, Kuźniarz-Rymarz S, et al. Dietary patterns as risk factors of differentiated thyroid carcinoma. Postepy Hig Med Dosw. 2012;66:11-15. doi: https://doi. org/10.5604/17322693.974647

20. Schwingshackl L, Hoffmann G. Does a Mediterraneantype diet reduce cancer risk?. Curr Nutr Rep. 2016;5:917. doi: https://doi.org/10.1007/s13668-015-0141-7

21. Haas P, Machado MJ, Anton AA, et al. Effectiveness of whole grain consumption in the prevention of colorectal cancer: meta-analysis of cohort studies. Int J Food Sci Nutr. 2009;60(Suppl 6):1-13. doi: https://doi. org/10.1080/09637480802183380

Recebido em 30/5/2020 Aprovado em 13/7/2020 